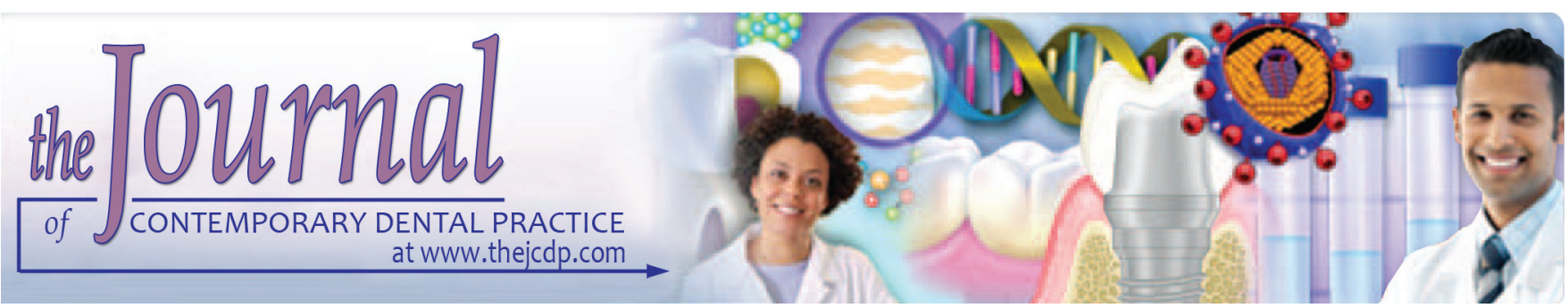

\title{
Variations in Flexural Strength of Heat-polymerized Acrylic Resin after the Usage of Denture Cleansers
}

\author{
${ }^{1}$ Mallikarjuna Ragher, ${ }^{2} \mathrm{G}$ Vinayakumar, ${ }^{3}$ Sanketsopan Patil, ${ }^{4}$ Aishwarya Chatterjee, ${ }^{5} \mathrm{DM}$ Mallikarjuna \\ ${ }^{6}$ Savita Dandekeri, ${ }^{7}$ V Swetha, ${ }^{8}$ MR Pradeep
}

\begin{abstract}
Objective: The objective of this study was to evaluate and compare changes in the flexural strength of heat-cured denture base resins when treated using denture cleansers.

Study design: A total of 40 specimens with dimension $65 \mathrm{~mm}$ length, $10 \mathrm{~mm}$ width, and $3 \mathrm{~mm}$ thickness were prepared as per ISO 1567 specification. A total of 10 specimens were immersed in distilled water to be used as control. Of the remaining 30 samples, 10 were treated with Clinsodent, 10 with VI-Clean, and 10 with Clanden denture cleansers. Specimens in each group were subjected to three-point flexural load in universal testing machine at a cross-head speed of $5 \mathrm{~mm} / \mathrm{min}$. The peak load $(\mathrm{N})$ was recorded and flexural strength was calculated. The findings were analyzed using Kruskal-Wallis analysis of variance and Mann-Whitney test.
\end{abstract}

Results: Heat-cured denture base resin selected for this study showed significant difference in flexural strength after immersion in denture cleansers Clinsodent, VI-Clean, and Clanden solutions, when compared with the control group.

Conclusion: Findings of this study showed that denture cleansers altered the flexural strength of heat polymerized acrylic resins that endured soaking cycles which simulated 180 days of use. Hence, denture cleansers should be used with caution, once a day after brushing the dentures. It is advisable for patients to follow the manufacturer's instructions.

1,5-7Department of Prosthodontics, Yenepoya Dental College Yenepoya University, Mangaluru, Karnataka, India

${ }^{2}$ Department of Prosthodontics, College of Dental Sciences Davanagere, Karnataka, India

${ }^{3}$ Department of Prosthodontics, Yogitha Dental College and Hospital, Ratnagiri, Maharashtra, India

${ }^{4}$ Department of Prosthodontics, SMS Medical College and Hospital, Jaipur, Rajasthan, India

${ }^{8}$ Department of Prosthodontics, AJ Institute of Dental Sciences Mangaluru, Karnataka, India

Corresponding Author: Mallikarjuna Ragher, Assistant Professor, Department of Prosthodontics, Yenepoya Dental College, Yenepoya University, Mangaluru, Karnataka, India Phone: +917676708707, e-mail: drmallis@gmail.com
Keywords: Denture base resins, Denture cleanser, Flexural strength.

How to cite this article: Ragher M, Vinayakumar G, Patil S, Chatterjee A, Mallikarjuna DM, Dandekeri S, Swetha V, Pradeep MR. Variations in Flexural Strength of Heat-polymerized Acrylic Resin after the Usage of Denture Cleansers. J Contemp Dent Pract 2016;17(4):322-326.

Source of support: Nil

Conflict of interest: None

\section{INTRODUCTION}

Polymethyl methacrylate was introduced in 1937 by Dr. Walter Wright and since then it has been successfully used for fabrication of denture bases, artificial teeth, and impression trays and many other applications in dentistry. However, it has always been masked by inherited strength characteristics, which includes poor fatigue resistance. ${ }^{1}$ The fracture of acrylic resin denture is a rather common occurrence and causes inconvenience. The fractures mainly occur as notches in midline area. In addition, fracture has been attributed to porosity, residual monomer, presence of cracks, and poor adoption of the removable prosthesis to the residual ridge. ${ }^{2}$

Home care instructions provided to patients during the denture placement appointments help in the maintenance of a healthy mucosa. Denture care is an indispensable element of general health, especially in elderly patients who cannot adequately brush their dentures because of disease, dementia, and poor dexterity. ${ }^{3}$

Continuous denture wearing leads to a higher prevalence of Mutans streptococci, Lactobacilli, Staphylococci, and yeasts in the oral cavity as compared with non-denturewearing subjects. Various methods have been evaluated in the literature for maintaining the denture hygiene; use of denture cleansers is one of the most commonly recommended methods. It is an efficacious method to 
prevent colonization of microorganism and denture plaque formation. However, certain studies have shown that immersion in certain cleansing solutions can affect the strength and the structure of denture base resins. If denture cleansers negatively affect the resins, decreasing the strength, greater incidence of denture fractures might occur, both outside and inside the mouth. Outside the mouth, failure occurs due to impact if the denture is dropped. Inside the mouth, occlusal forces may cause fractures. Midline fracture of the denture, for example, may occur as a consequence of flexural fatigue, resulting from cyclic deformation of the base during function. ${ }^{4}$

An extensive literature review reveals a difference in opinion as to whether immersion in denture cleansers adversely affects the strength property of denture base resins. As of now, limited literature is available to evaluate the effects of denture cleansers on flexural strength of denture base resins that are commercially available in India.

The purpose of the present study was to investigate the effects of certain brands of denture cleansers on the flexural strength of denture base resin used in India.

\section{METHODOLOGY}

\section{Fabrication of Master Die for Preparation of Study Mold}

For the study, a precise, metal mold was custom fabricated at Bapuji Institute of Engineering and Technology, Davangere, measuring $65 \times 10 \times 3 \mathrm{~mm}$ to make test samples (Fig. 1).

\section{Preparation of Dental Plaster Mold Space}

Wax patterns were obtained by pouring wax into the die space present within the metal mold. The wax patterns obtained were checked for voids and inaccuracies. The wax patterns were then invested in a dental flask (Kavo,

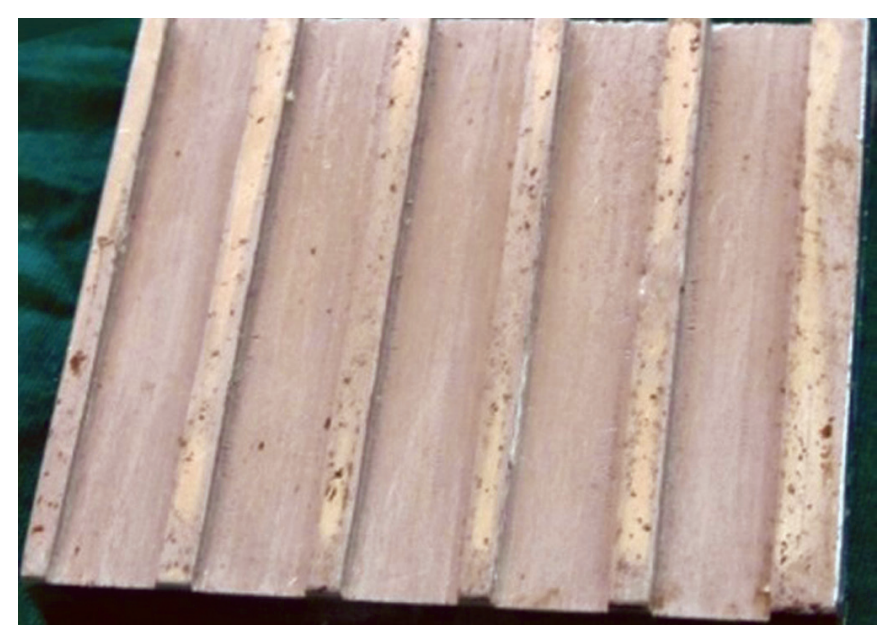

Fig. 1: Metal mold used for fabrication of wax patterns
Germany) using dental plaster (VIP chemicals, India) following the manufacturer's instructions for waterpowder ratio, mixing time, and setting time. Mechanical vibrator was used to prevent air trapping during investing. Once the plaster was completely set, dewaxing was done for 10 minutes. The mold space thus obtained was used for the preparation of the test samples.

\section{Preparation of Test Samples}

Separating medium (cold mold seal, DPI) was applied on to the dental plaster mold with the help of a camel hair brush and dried. The denture base resin (Trevalon, Dentsply, Gurgaon, India) material was mixed according to manufacturers' instructions and packed at dough stage. Powder-liquid ratio for Trevalon denture base material (conventional type) is $24 \mathrm{gm} / 10 \mathrm{ml}$. The specimens were bench cured for 1 hour and polymerized according to manufacturers' instructions. After processing, the specimens were bench cooled for 1 hour and then finished with various grits of sand paper $(80,320,400,1,000)$ followed by polishing with pumice. Thickness, length, and width of each specimen were verified with an electronic digital caliper (accurate up to $\pm 0.02 \mathrm{~mm} / 0.001$ inch; Zoom, India). The specimens were stored in distilled water for 24 hours.

\section{Immersion Procedure}

Specimens were randomly divided into four groups and immersed in the respective denture cleanser according to manufacturers' instructions (Fig. 2). Clinsodent (ICPA) - one $480 \mathrm{mg}$ tablet was diluted in $50 \mathrm{ml}$ of warm water and immersed for 30 minutes; VI-Clean (Vishal Pharmaceuticals, Ahmedabad) - one teaspoonful of liquid denture cleanser was diluted in $50 \mathrm{ml}$ of water and immersed for 30 minutes; Clanden - one $480 \mathrm{mg}$ tablet was diluted in $50 \mathrm{ml}$ of water and immersed for 3 minutes. The capacity of the container in which specimens were stored was $400 \mathrm{ml}$. Hence eight tablets of Clinsodent and Clanden and eight teaspoonfuls of VI-Clean were diluted in $400 \mathrm{ml}$ of water and samples were immersed in it. After immersion, the resin specimens were removed from the

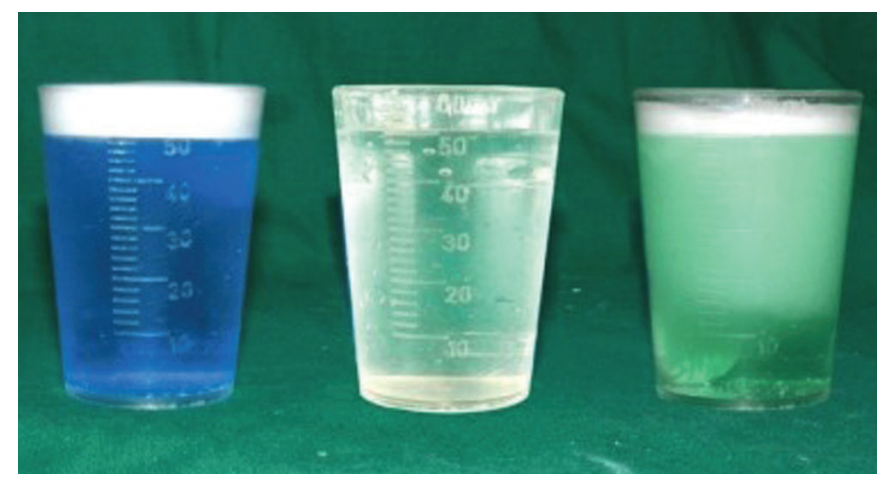

Fig. 2: Clinsodent, VI-Clean, and Clanden denture cleanser in measuring jar 
cleanser solutions, thoroughly washed in running water, dried with absorbent paper, and then the procedure of immersion was repeated. This procedure was performed for a total of 30 times over a period of 6 days simulating 180 days of cleansing by the patient.

\section{Testing the Flexural Strength}

Testing was done by using a universal testing machine (Model H5KS, Tinius Olsen, USA). A total of 10 specimens of each group were used for flexural strength determination $(n=40)$ (Fig. 3). All the specimens were marked in the center. The customized jig was positioned on the universal testing machine and the specimens were loaded one at a time. Load was applied at the center of the specimen at a cross-head speed of $5 \mathrm{~mm} / \mathrm{min}$, until it fractured. The machine has a digital monitor that indicates the amount of force being applied to the test specimen. When the specimen breaks, the reading on the digital scale automatically stops, thus indicating the fracture load. The load at which fracture occurred was noted and the transverse strength was calculated using the following formula:

$$
\sigma=\frac{3 \mathrm{LP}}{2 \mathrm{WT}^{2}}
$$

where $\mathrm{P}=$ fracture load, $\mathrm{L}=$ the distance between the supports $(50 \mathrm{~mm}), \mathrm{W}=$ specimen width $(10 \mathrm{~mm})$, and $\mathrm{T}=$ specimen thickness $(3 \mathrm{~mm})$

\section{Statistical Analysis}

Data collected by experiments were computerized and analyzed using the Statistical Package for Social Sciences (SPSS) version 17.0. Results were expressed in frequencies. Nonparametric tests, namely Kruskal-Wallis analysis of variance (ANOVA) test and Mann-Whitney U test, were used for testing the statistical significance. For all tests, a p-value of 0.05 or less was considered statistically significant.

\section{RESULTS}

Table 1 and Graph 1 represent the mean and standard deviation of flexural strength of the Clinsodent, VI-Clean,

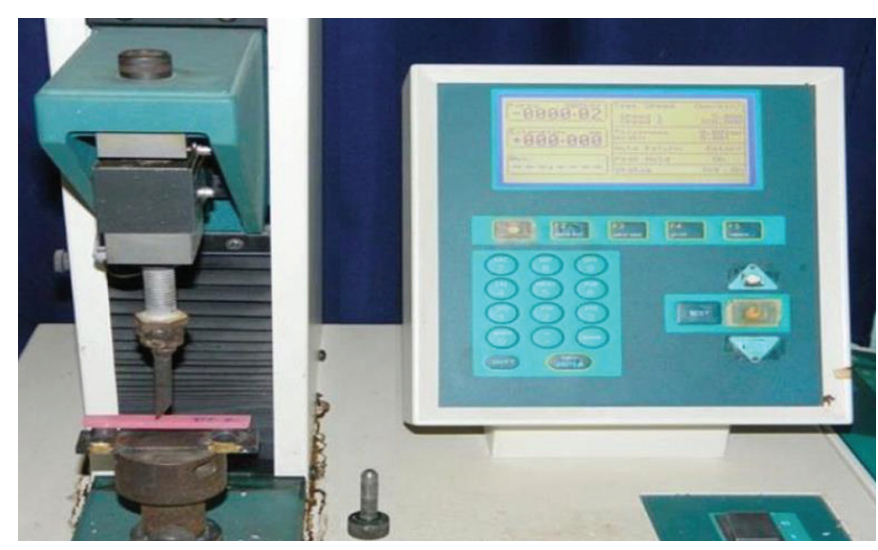

Fig. 3: Position of the sample in a universal testing machine and Clanden groups compared with the control group. In Table 2, the significant differences can be observed in the mean values of flexural strength for all the groups after immersion in denture cleansers following the 180-day simulation. As shown in Table 3, the specimens that were immersed in Clinsodent denture cleansers showed a significantly higher reduction in flexural strength followed by Clanden and VI-Clean denture cleansers compared with the control group.

\section{DESCRIPTIVE STATISTICS}

Table 1: Descriptive statistics of flexural strength with mean and standard deviation of the Clinsodent, VI-Clean, and Clanden groups compared with the control group

\begin{tabular}{lllll}
\hline & & & \multicolumn{2}{c}{ Flexural strength (MPa) } \\
\cline { 5 - 5 } SI. no. & Group & $\begin{array}{l}\text { Number of } \\
\text { specimens }\end{array}$ & Mean & $\begin{array}{l}\text { Standard } \\
\text { deviation }\end{array}$ \\
\hline 1 & Control & 10 & 111.02 & 11.33 \\
2 & Clinsodent & 10 & 71.467 & 9.75 \\
3 & VI-Clean & 10 & 91.79 & 7.50 \\
4 & Clanden & 10 & 81.31 & 11.83 \\
\hline
\end{tabular}

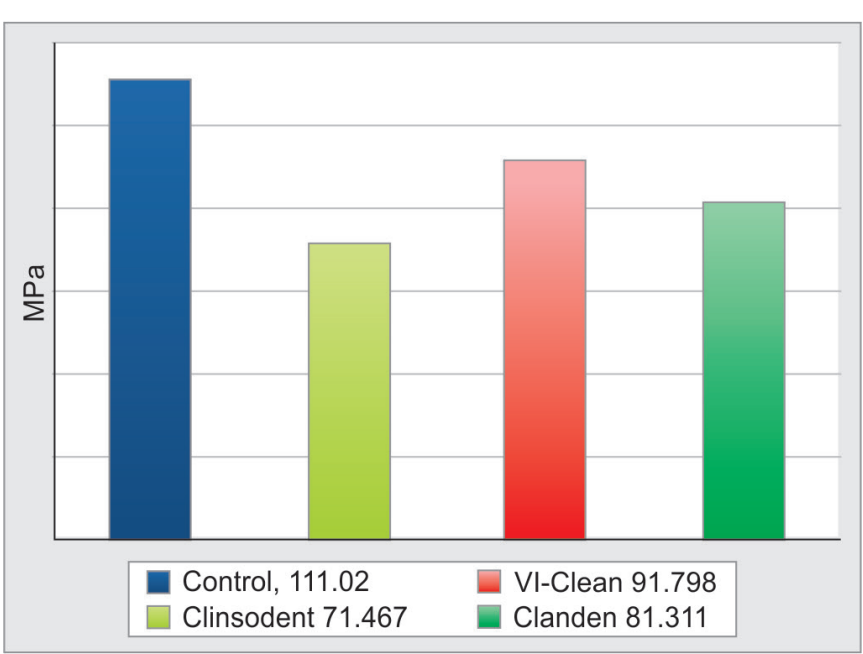

Graph 1: Comparision of mean flexural strength of various groups tested

Table 2: Kruskal-Wallis (ANOVA) used to analyze the total flexural strength data

\begin{tabular}{lll}
\hline & $\chi^{2}$ value & $p$-value \\
\cline { 2 - 3 } Between groups & 27.24 & 0.000 \\
\hline
\end{tabular}

Table 3: Descriptive statistics and intergroup comparison of flexural strength

\begin{tabular}{llll}
\hline & Mann- & & \\
& Whitney & p-value & Significance \\
\hline Control group and Clinsodent & 0.000 & 0.000 & $\mathrm{HS}$ \\
Control group and VI-clean & 10.00 & 0.002 & $\mathrm{HS}$ \\
Control group and Clanden & 2.500 & 0.000 & $\mathrm{HS}$ \\
Clinsodent and VI-clean & 3 & 0.000 & $\mathrm{HS}$ \\
Clinsodent and Clanden & 25.00 & 0.059 & $\mathrm{NS}$ \\
VI-clean and Clanden & 24.00 & 0.049 & $\mathrm{~S}$ \\
\hline
\end{tabular}

HS: Highly Significant; NS: Not Significant; S: Significant 


\section{DISCUSSION}

Denture cleansers are widely used to prevent colonization by Candida albicans and related Candida species and to prevent denture plaque formation. Commercial denture cleansing products fall essentially into two classes of immersion cleansers:

- Alkaline peroxide

- Alkaline hypochlorites

Alkaline peroxides, such as Efferdent, Polident, Clinsodent, and Clanden (the last two used in the present study), work basically through an oxygen-liberating mechanism that purportedly loosens debris and removes light stain. They have pleasant odor and show little reported harmful effect on the metal components of partial dentures. They do not appear to remove calculus or heavy staining, however.

Alkaline hypochlorite denture cleanser solutions, such as Mersene and VI-Clean liquid denture cleanser (used in the present study), work basically by removing light stains and food debris with a bleaching action. While several studies have supported the superior plaqueremoving abilities of these products, their limited removal of calculus and the corrosive effects on aluminum-based dentures and cobalt chrome alloys remain drawbacks to effective long-term use. ${ }^{5}$ Literature states that daily use of denture cleansers can affect the physical and mechanical properties of denture base materials. ${ }^{6}$

In the present study, the flexural strength of heat-cured denture base resins was evaluated and compared after immersing them in three types of commercially available denture cleansers.

Results of the present study showed that flexural strength of the denture base resin significantly decreased after immersion in the denture cleanser solutions Clinsodent, VI-Clean, and Clanden when compared with the control group. It may be because chemical interactions of denture cleanser with methyl methacrylate have an influence on the bond strength of denture base resins.

The results are in correlation with an in vitro study by Peracini et al. ${ }^{3}$ They evaluated the effects of denture cleansers (Corega tabs, Bony Plus) on color change, surface roughness, and flexural strength of heat-polymerized acrylic resin and concluded that the color changes were significantly higher for Corega tabs as compared with the control group. Bony Plus had a significantly higher surface roughness than the other groups. Corega tabs and Bony Plus groups presented significantly lower flexural strength than the control group.

Similar results were found by Robinson et al, ${ }^{7}$ who conducted a series of tests that were devised to assess the effect of subjecting acrylic resin denture materials to denture cleanser at recommended and elevated temperatures. The results concluded that heat-cured specimens that had whitened also suffered a reduction in flexural strength and microscopy, indicating changes in the interstitial matrix of the two-phase structure of the resins.

Subsequent studies done by Crawford et $\mathrm{al}^{8}$ and also concluded that heightened temperature beyond manufacturers' recommendation caused a decline in the flexural strength of denture base resin over repeated exposures. The studies emphasized that though the use of denture cleansers was a safe method for disinfection of dentures, the guidelines followed by the manufacturer should be followed. ${ }^{9}$

The results are in correlation with another in vitro study by Neppelenbroek et al, ${ }^{9}$ who demonstrated a significant reduction in the mean hardness value for the denture base resin tested with sodium perborate solution. However, the flexural strength results comply with the minimum value (65 $\mathrm{MPa}$ ) set forth by ADA specification no. 12 , and it may have no clinical relevance. ${ }^{10}$

A similar study conducted by Garcia et al evaluated the effects of denture cleansers on the surface hardness and surface roughness of the denture base resin, $\mathrm{Co}-$ $\mathrm{Cr}$ and $\mathrm{Ti}-6 \mathrm{Al}-4 \mathrm{~V}$ alloys. They found that cleanser manipulated using sodium perborate increased the surface roughness and hardness of $\mathrm{Co}-\mathrm{Cr}$ and Ti-6Al-4V alloys as well as denture base resins due to its incapacity to remove the pellicle formed on the acrylic resin and dental alloys. ${ }^{11}$

On the contrary, Sato et $\mathrm{al}^{4}$ assessed the flexural strength and color alteration of acrylic resin, immersed in denture cleansers for different periods of time. They found no significant differences in any of the denture cleansers (Bony Plus, Corega tabs, Efferdent Plus, and control) or between the soaking periods throughout the soaking cycles simulating 30 days of use. This is due to the use of 30 days' simulating effect when compared with the present study using 180 days' simulating effect.

Devlin and Kaushik ${ }^{12}$ showed water absorption on acrylic surfaces caused by peroxide solution, which results in irreversible damage to the mechanical properties of denture base resin. The water sorption by acrylic resins causes dimensional instability and fatigue, which can lead to crack formation and, subsequently, to fracture of the denture. ${ }^{13}$

Another factor involved in the mechanical properties is the residual methyl methacrylate monomer in the polymerized acrylic resin, which has a plasticizing effect. ${ }^{14}$ The specimen of this study was immersed in distilled water for residual monomer elimination. ${ }^{10}$

These results should be clinically interpreted with caution, because different results may be obtained when 
fatigue stress during function (in vivo) is combined with the chemical action of denture cleansers. If denture cleansers lead to a reduction in strength, a higher incidence of denture fractures could occur.

\section{Limitations of the Study}

In this study, specimens were prepared in accordance with ISO 1567 (1999) specification. Though the study was carried out with utmost accuracy, it has certain limitations, which are listed below:

- In clinical situations, a uniform thickness of denture base resin may not be $3 \mathrm{~mm}$, as used in this study.

- In spite of following the standard and uniform protocol for preparing, curing, and finishing all specimens, the homogeneity of mix, presence of internal porosities, and release of stress during finishing and polishing procedures could not be controlled.

- In the oral cavity, denture bases are exposed to varying magnitudes acting in different directions. The same situation could not be simulated in this in vitro study.

\section{Scope for Further Study}

- Chemical interaction of denture cleansers on the bond strength of polymethyl methacrylate should be evaluated.

- The testing period should be longer for the simulation of long-term use, and association with mechanical cleaning methods could show potential interaction.

- Research with biofilm could influence the hygiene solutions.

- Other conditions of oral environment should be simulated, such as continuous cyclic loading.

\section{CONCLUSION}

Within the limitations of the study, the following conclusions were drawn:

- Immersion in denture cleanser solutions Clinsodent, VI-Clean, and Clanden showed significant changes in the flexural strength of heat polymerized acrylic resins.
- Clinsodent shows more reduction in the flexural strength followed by Clanden and VI-Clean denture cleansers.

\section{REFERENCES}

1. Gutteridge DL. The effects of including ultra-high modulus polyethylene fiber on the impact strength of acrylic resins. Br Dent J 1988 Mar 19;164(6):177-180.

2. Faot F, Costa MA, Del Bel Cury AA, Rodrigues Garcia RC. Impact strength and fracture morphology of denture acrylic resins. J Prosthet Dent 2006 Nov;96(5):367-373.

3. Peracini A, Davi LR, de Queiroz Ribeiro N, de Souza RF, Lovato da Silva $\mathrm{CH}$, de Freitas Oliveira Paranhos H. Effect of denture cleansers on physical properties of heat-polymerized acrylic resin. J Prosthodont Res 2010 Apr;54(2):78-83.

4. Sato S, Cavalcante MR, Orsi IA, Paranhos Hde F, Zaniqeilli $\mathrm{O}$. Assessment of flexural strength colour alteration of heat polymerized acrylic resin after simulated use of denture cleanser. Braz Dent J 2005;16(2):124-128.

5. Abelson DC. Denture plaque and denture cleansers. J Prosthet Dent 1981 Apr;45(4):376-379.

6. Backenstose WM, Wells JG. Side effects of immersion type cleansers on the metal components of dentures. J Prosthet Dent 1977 Jun;37(6):615-621.

7. Robinson JG, McCode JF, Store R. Denture base: the effect of various treatments on clarity, strength and structure. J Dent 1987 Aug;15(4):159-165.

8. Crawford CA, Lloyd CH, Newton JP, Yemm R. Denture bleaching: a laboratory simulation of patients cleaning procedure. J Dent 1986 Dec;14(6):258-261.

9. Neppelenbroek KH, Pavarina AC, Vergani CE, Giampaolo ET. Hardness of heat-polymerized acrylic resins after disinfection and long-term water immersion. J Prosthet Dent 2005 Feb;93(2):171-176.

10. Revised American Dental Association specification no. 12 for denture base polymers. J Am Dent Assoc 1975 Feb;90(2): 451-458.

11. Rodrigues Garcia RC, Joane Augusto de S Jr, Rached RN, Del Bel Cury AA. Effect of denture cleansers on the surface roughness and hardness of a microwave-cured acrylic resin and dental alloys. J Prosthodont 2004 Sep;13(3):173-178.

12. Devlin H, Kaushik P. The effect of water absorption on acrylic surface properties. J Prosthodont 2005 Dec;14(4):233-238.

13. Beyli MS, von Fraunhofer JA. An analysis of causes of fracture of acrylic resin dentures. J Prosthet Dent 1981 Sep;46(3):238-241.

14. Doğan A, Bek B, Cevik NN, Usanmaz A. The effect of preparation conditions ofacrylic denture base materials on the level of residual monomer, mechanical properties and water absorption. J Dent 1995 Oct;23(5):313-318. 